\title{
Hemoptisis de etiología desconocida. Informe de caso
}

\author{
Hemoptysis of unknown etiology. Case report
}

Carlos Raúl Santa María-Ramírez; Kellyta Christinne Silva da Mata; Daniela da Mata Queiroz; Raquel Yuska Dantas ${ }^{1}$; Shirley Galeano Reynal ${ }^{2}$

\section{RESUMEN}

Introducción: La hemoptisis es una cantidad variable de sangre que pasa por la glotis proveniente de las vías aéreas y de los pulmones.

Caso Clínico: Hombre, 32 años, obeso, tabaquista, ingresó por disnea de moderados a mínimos esfuerzos de 4 meses de evolución. El paciente refirió tos con expectoración hemoptoica de 05 años de evolución, predominio nocturno, constatada al despertar. Refirió haberse sometido a varios procedimientos quirúrgicos: por sospecha de torsión testicular, lamectomía descompresiva y extracción de aguja de costura en región parietal posterior a una TAC por episodios tónico-clónicos. Durante su internación, se le realizaron varios exámenes todos sin éxito. Un día, se identificó actitud sospechosa, comprobándose la ingesta de sangre del conducto de poliflex, la cual colaboró con el diagnóstico definitivo de "síndrome facticio".

Discusión: Como parte de la salud integral no debemos olvidar la evaluación de la salud mental y hacer más hincapié en la historia clínica.

Palabras clave: Hemoptisis; Síndrome bronquial obstructiva; Trastorno facticio.

Fecha de recepción: junio 2020; fecha de aceptación: julio 2020

${ }^{1}$ Universidad Privada María Serrana, Carrera de Medicina. Asunción, Paraguay

${ }^{2}$ Universidad Privada María Serrana, Coordinación de Investigación y Extensión de la Carrera de Medicina. Asunción, Paraguay

Autor de correspondencia: Carlos Raúl Santa María-Ramírez. Email: krlitoz26@gmail.com 


\section{ABSTRACT}

Introduction: Hemoptysis is a variable amount of blood that passes through the glottis from the airways and lungs.

Clinical Case: Man, 32 years old, obese, tobacco user, admitted for dyspnea of moderate to minimal efforts of 4 months of evolution. The patient reported a cough with hemoptoic expectoration of 05 years of evolution, predominantly at night, confirmed upon awakening. He reported having undergone several surgical procedures: on suspicion of testicular torsion, decompressive lamectomy and removal of a sewing needle in the parietal region after $\mathrm{CT}$ for tonic-clonic episodes. During his hospitalization, he underwent several examinations, all without success. One day, a suspicious attitude was identified, checking the blood intake of the poliflex duct, which collaborated with the definitive diagnosis of "factitious syndrome".

Discussion: As part of comprehensive health, we must not forget the evaluation of mental health and place more emphasis on medical history.

Keywords: Hemoptysis; Obstructive bronchial syndrome; Factitious disorder. 


\section{INTRODUCCIÓN}

La hemoptisis es una cantidad variable de sangre que pasa por la glotis proveniente de las vías aéreas y de los pulmones (1).

La Hemorragia pulmonar es la presencia de fluido hemorrágico (color "rojo fresco") en la tráquea, acompañado de una descompensación respiratoria aguda con aumento de los requerimientos de oxígeno y/o del soporte ventilatorio (2).

Las infecciones son la causa más frecuente. Presentamos el caso de un paciente adulto joven con hemoptisis leve crónica, internado durante un mes, con búsquedas activas de sitio de sangrado y múltiples análisis laboratoriales.

\section{Descripción del Caso Clínico}

Nombre: J.G.

Sexo: Masculino

Edad: 32 años

Estado civil: Unión libre

Profesión: Zarandeador de carbón desde los 11 a 19 años.

Asistente de cocina durante 7 años

Vendedor de ropa hasta la actualidad

Procedencia: Capiatá

Fecha ingreso: 22/06/2017

Fecha egreso: 30/07/2017

Motivo consulta: Dificultad respiratoria

\section{- Antecedentes de enfermedad actual}

Tos con expectoración hemoptoica, de 5 años de evolución, en moderada cantidad, de predominio nocturno, con episodios mensuales de hemoptisis leve 10cc aproximadamente que se constataban al despertar por manchar la ropa de cama.

Dificultad respiratoria de moderados a mínimos esfuerzos, que mismo con reposo de 4 meses de evolución se acompaña de dolor tipo puntada en región posterolateral del hemitórax izquierdo, accesos de tos varias veces al día y con aumento del volumen de la hemoptisis de aproximadamente 50 cc con presencia de coágulos.
Niega pérdida de peso y fiebre.

\section{- Antecedentes remotos de la enferme-} dad actual

07/07/2011 - Dolor testicular intenso, con cirugía exploradora que se descarta torsión del cordón espermático. 09/092011 - Laminectomía descompresiva. Hace 5 meses acudió a la urgencia siendo encaminado a la UTI por convulsiones tónico-clónicas, realizando una TAC de cráneo que se observó una imagen metálica en región parietal, siendo sometido a un procedimiento para extracción de una aguja de costura.

Hace 2 meses presentó rectorragia y se le realizó una colonoscopia que diagnosticó hemorroides grado I, no complicada.

\section{- Antecedentes patológicos actual}

Sibilancias desde hace 5 años aproximadamente, que sigue sin tratamiento, negando episodios en la infancia.

Hábitos tóxicos: tabaquista 25.5 paquetes al año, hasta la actualidad, bebedor social y niega consumo de drogas.

\section{- Antecedentes patológicos familiar}

Madre encamada con lesiones eritematosas en ambas piernas, obesa mórbida que no cuenta con seguimiento médico. Tío materno fallecido a los 30 años, causa no filiada, con cuadro de hemoptisis y rectorragia. Padre no mantiene contacto con el paciente aproximadamente hace 15 años.

\section{- Examen físico}

Signos Vitales:

$\begin{array}{ll}\text { - } & \text { PA: } 120 / 80 \\ \text { - } & \text { FC: } 77 \times \text { min } \\ \text { - } & \text { FR: } 18 \times \text { min } \\ \text { - } & \text { T: } 36^{\circ} \mathrm{C}\end{array}$

Otros datos de relevancia:

- $\quad$ Sat. O2: 98\% AA

- Peso: $112 \mathrm{Kg}$

- Talla: $178 \mathrm{~cm}$

- $\quad$ IMC: 35,4 


\section{- Ectoscopia 22/06/2017}

Se constató que el paciente es obeso (grado I), normosómico, cuya edad cronológica no coincidió con la biológica, adopta en el lecho decúbito dorsal indiferente, facies dolorosas.

Portaba vía venosa periférica en brazo derecho.

Impresiona patología crónica agudizada.

\begin{tabular}{|l|l|}
\hline Aparato & Examen fisico \\
\hline Boca & $\begin{array}{l}\text { Mucosa húmeda, dientes en mal } \\
\text { estado con bordes cortantes }\end{array}$ \\
\hline Cardiovascular & $\begin{array}{l}\text { R1 y R2 normofonéticos, con } \\
\text { ausencia de soplos y golpes, ictus } \\
\text { cordis no desplazado }\end{array}$ \\
\hline Respiratorio & $\begin{array}{l}\text { Dolor en región lateral izquierda, } \\
\text { cicatriz vertical de 5cm de longitud } \\
\text { en región posterior del tórax, MV } \\
\text { disminuido globalmente, sibilancias } \\
\text { aisladas en campo medio pulmonar } \\
\text { izquierdo }\end{array}$ \\
\hline Abdomen & $\begin{array}{l}\text { Blando, depresible, no doloroso, } \\
\text { RH + conservados, no } \\
\text { visceromegalias }\end{array}$ \\
\hline Osteoarticular & $\begin{array}{l}\text { Sin deformidades, articulaciones } \\
\text { simétricas, no dolorosas }\end{array}$ \\
\hline Piel y mucosa & $\begin{array}{l}\text { Múltiples tatuajes en ambos brazos. } \\
\text { Coloración de piel y mucosas } \\
\text { conservadas }\end{array}$ \\
\hline SNC & Glasgow 15/15 \\
\hline
\end{tabular}

\section{Examenes Laboratoriales 22/06/2017}

\begin{tabular}{|lcc|l|}
\hline \multicolumn{2}{|l|}{ Glóbulos Blancos: } & $8340 / \mu \mathrm{L}$ & \multicolumn{1}{|c|}{ ORINA SIMPLES } \\
\hline Neutrófilos: & 4930 & $64 \%$ & $\mathrm{PH}: 5.0$ \\
\hline Linfocitos: & 2410 & $30 \%$ & Proteínas: negativo \\
\hline Monocitos: & 586 & $3 \%$ & Sangre: negativo \\
\hline Eosinófilos: & 356 & $3 \%$ & Nitritos: negativo \\
\hline Eosinófilos: & 356 & $3 \%$ & Cetonas: negativo \\
\hline Hemoglobina: $15.8 \mathrm{~g} / \mathrm{dl}$ & Urobilinogeno: negativo \\
\hline Hematocrito: & $44,9 \%$ & Leucocitos: 1 -2 / campo \\
\hline VSG: & $5 \mathrm{~mm}$ & Hematies: $0-2$ / campo \\
\hline PCR: & $\leq 6$ & \\
\hline
\end{tabular}




\begin{tabular}{|l|l|l|}
\hline Gasometria & Resultado & Rango \\
\hline PH & 7.4 & $7.36-7.45$ \\
\hline PCO2 & 37 & $35-45$ \\
\hline PO2 & 73 & $80-100$ \\
\hline HCO3 & 22 & $22-26$ \\
\hline TCO2 & 27 & $23-27$ \\
\hline ABE & -2 & $-2.4-2.4$ \\
\hline SBE & -2 & \\
\hline SBC & 23 & $22-26$ \\
\hline SatO2 & 94 & $95-99$ \\
\hline
\end{tabular}

\begin{tabular}{|l|l|l|}
\hline Estudio & Resultado & Rango de referencia normal \\
\hline Creatinina & 0.85 & $0.65-1.3 \mathrm{mg} / \mathrm{dl}$ \\
\hline Urea & 18 & $15-55 \mathrm{mg} / \mathrm{dl}$ \\
\hline Sodio & 138 & $136-145 \mathrm{mEq} / \mathrm{dl}$ \\
\hline Potasio & 4.2 & $3.5-5.1 \mathrm{mEq} / \mathrm{dl}$ \\
\hline Cloro & 104 & $98-107 \mathrm{mEq} / \mathrm{dl}$ \\
\hline Magnesio & 2.0 & $1.9-2.5 \mathrm{mg} / \mathrm{dl}$ \\
\hline Proteinas totales & 6.9 & $6.0-8.7 \mathrm{~g} / \mathrm{dl}$ \\
\hline Albumina & 4.5 & $>$ a $3.5 \mathrm{~g} / \mathrm{dl}$ \\
\hline Bilirrubina total & 0.84 & $0.0-1.0 \mathrm{mg} / \mathrm{dl}$ \\
\hline AST & 33 & $0.031 .0 \mathrm{mg} / \mathrm{dl}$ \\
\hline ALT & 34 & $0.0-37.0 \mathrm{mg} / \mathrm{dl}$ \\
\hline $\begin{array}{l}\text { Fosfatasa } \\
\text { alcalina }\end{array}$ & 102 & $40-150 \mathrm{U} / \mathrm{L}$ \\
\hline
\end{tabular}

\begin{tabular}{|ll|}
\hline Tiempo de Protrombina: & $12 \mathrm{seg}$ \\
\hline \% de Actividad de Protrombina: & $104 \%$ \\
\hline Fibrinógeno: & $379 \mathrm{mg} / \mathrm{dl}$ \\
\hline
\end{tabular}

\begin{tabular}{|l|l|}
\hline Estudio & Resultado \\
\hline VIH & Negativo \\
\hline VDRL & Negativo \\
\hline
\end{tabular}

\begin{tabular}{|l|l|}
\hline Perfil Reumatológico & Resultado \\
\hline ANA & Negativo \\
\hline AntiDNA & Negativo \\
\hline C3 & $141 \quad / \quad 82-185$ \\
\hline C4 & $30.6 / 15-53$ \\
\hline FR & $\leq 8 \quad / \quad 0-8$ \\
\hline
\end{tabular}




\begin{tabular}{|l|l|}
\hline Estudio & Resultado \\
\hline ANCA-C & Negativo \\
\hline ANCA-P & Negativo \\
\hline
\end{tabular}

\section{Exámenes de imagen}

\begin{tabular}{|c|}
\hline ECG \\
\hline Ritmo Sinusal \\
\hline Ecocardiograma \\
\hline $\begin{array}{l}\text { - Válvulas estructuralmente normales } \\
\text { - Presión pulmonar normal } \\
\text { - FE: } 70 \%\end{array}$ \\
\hline TACAR de tórax \\
\hline $\begin{array}{l}\text { - Normal } \\
\text { - Estructuras conservadas } \\
\text { - Sin derrame y engrosamiento pleural } \\
\text { - Parénquima conservado } \\
\text { - No presentó bullas, dilataciones, bronquiectasias y cavernas }\end{array}$ \\
\hline
\end{tabular}

\section{Exámenes de cultivo}

\begin{tabular}{|l|l|l|}
\hline Estudio & Resultado & Cultivo \\
\hline $\begin{array}{l}\text { Esputo } \\
\text { 24/06/2017 }\end{array}$ & $\begin{array}{l}\text { Frotis para BAAR } \\
\text { negativo }\end{array}$ & $\begin{array}{l}\text { Pendiente para } \\
\text { tuberculosis. Hongos } \\
\text { negativo }\end{array}$ \\
\hline $\begin{array}{l}\text { Esputo } \\
\text { 26/O6/2017 }\end{array}$ & $\begin{array}{l}\text { Frotis para BAAR } \\
\text { negativo }\end{array}$ & $\begin{array}{l}\text { Pendiente para } \\
\text { tuberculosis. Hongos } \\
\text { negativo }\end{array}$ \\
\hline $\begin{array}{l}\text { Esputo } \\
\text { 01/07/2017 }\end{array}$ & $\begin{array}{l}\text { Genexpert } \\
\text { negativo }\end{array}$ & $\begin{array}{l}\text { Pendiente para } \\
\text { tuberculosis. }\end{array}$ \\
\hline BAL 06/07/2017 & $\begin{array}{l}\text { Microbiota } \\
\text { nabitual de VAS }\end{array}$ & $\begin{array}{l}\text { Pendiente para } \\
\text { tuberculosis. }\end{array}$ \\
\hline
\end{tabular}

\section{Otros exámenes complementarios}

\begin{tabular}{|l|l|}
\hline Estudio & Informe \\
\hline FBC $29 / 06 / 2017$ & Sin particularidades \\
\hline FBC $06 / 07 / 2017$ & Sin particularidades \\
\hline RNC anterior $10 / 07 / 2017$ & Sin particularidades \\
\hline NFC $13 / 07 / 2017$ & Sin particularidades \\
\hline FBC $13 / 07 / 2017$ & Sin particularidades \\
\hline NFC $16 / 07 / 2017$ & Sin particularidades \\
\hline EDA $17 / 07 / 2017$ & Sin particularidades \\
\hline
\end{tabular}


- AngioTAC

Sin particularidades, sin malformaciones, no se observó tromboembolismo, ni signos de lesión vascular.

\section{Diagnósticos probables:}

- Síndrome bronquial obstructivo de etiología a filiar,

- Tuberculosis

\section{Tratamiento recibido}

- $\quad$ Ampicilina IBL 6 g/d x 21 días

- Ciprofloxacina 400mg/d x 19 días

- Codeína gotas: 30 gotas c/8hs.

- Salbutamol/Ipatropio $100 \mu \mathrm{g}$ :

2puff x AC s/Necesidad

- $\quad$ Salmeterol / Fluticazona 250 $\mu \mathrm{g}: 2$ puff $\mathrm{x}$ AC c/12hs.

- Hidrocortisona $300 \mathrm{mg} / \mathrm{d}$ x 10 días

- Prednisona 40mg/d en descenso gradual hasta 0

\section{Evolución de la enfermedad}

20:00 - paciente se encontraba en su unidad higienizado y medicado por el servicio de enfermería.

20:30 - paciente fue al baño, retornando a las 20:45 horas.

21:00 - llamó al auxilio por los familiares, donde se observó hemoptisis masiva a cuál fue realizado una estabilización y revisión de las vías aéreas. Paciente refiero cansancio sin pérdida del conocimiento y signos en rango.

21:10 - se recibió llamado del servicio de enfermería, donde se observó en el piso del baño sangre del conducto del equipo de poliflex conectado a la vía venosa periférica cargado de sangre, siendo que el licenciado había registrado la limpieza de la conexión y no se pudo refluir la sangre por los conductos.

Por la actitud sospechosa del paciente se solicitó evaluación del servicio de psiquiatría, constatando la posibilidad de que el paciente podría presentar el síndrome facticio o síndrome de Munchausen.

\section{El síndrome Facticio}

Dicho síndrome se clasifica como un trastorno en el que existe una fabricación o inducción consciente o intencional de signos y síntomas de un trastorno físico, emocional o cognitivo, e incluso las pruebas de laboratorio pueden ser manipuladas (3).

El síndrome de facticio también se ha denominado "síndrome de Munchausen", "adicción al hospital", "adicción polisúrgica" y "síndrome del paciente profesional"(4).

El trastorno facticio no tiene una prevalencia conocida, sin embargo, se estima que aproximadamente el $1 \%$ de los casos de personas hospitalizadas cumplen con los criterios de diagnóstico(5). Este trastorno, aunque de baja ocurrencia, tiene un alto impacto en la vida del sujeto afectado y de quienes lo rodean, así como en los altos costos de los sistemas que lo atienden, ya que estos estos pacientes a menudo migran entre los servicios de salud(6). Tal aumento de costos se genera principalmente debido a exámenes y procedimientos innecesarios y no concluyentes, tratamiento de diagnóstico insuficiente y diagnóstico erróneo que pueden surgir durante la interacción con esta población, estadía prolongada en el seguimiento profesional porque no hay un diagnóstico y desarrollo concreto.

\section{DISCUSIÓN}

Cuando un paciente llega a la consulta, su historia clínica nos da información de primera mano sobre su estado de salud y sus patologías previas. Información que resul- ta muy útil ya que permite al médico conocer varias cosas como las dolencias previas que probablemente expliquen los síntomas del paciente, orientar el diagnóstico, enca- 
minar al tratamiento adecuado, tener una visión general del estado del paciente y su evolución a lo largo del tiempo, presumiendo sobre posibles consecuencias y demás.

Como parte de la salud integral no debemos olvidar la evaluación de la salud mental.

Los gastos de insumos, el costo de los procedimientos y el tiempo invertido en un paciente en el que la clínica siempre fue soberana, nos debe ayudar en la autocrítica, no valoramos la historia clínica de forma correcta, olvidando que ésta nos otorga la oportunidad de crear diagnósticos sindromáticos.

\section{REFERENCIAS}

1. Jougon J BDFMBTVPGFea. Massive Hemoptysis: what place for medical and surgical treatment. Eur J Cardiothorac Surg. 2002;22(3): 345-51.

2. Cloherty et al. Pulmonary Hemorrage. Manal of Neonatal Care. 2004;(7).

3. Pereira Aline Viana MBCGGMRGBNG. Transtorno Factício e a Equipe interdisciplinar: identificação de sinais e fatores de risco. CoDAS. 2019 Feb.

4. (OMS). Omdls. La clasificación CIE-10 de los trastornos Mentales y de comportamiento. Criterios de diagnósticos
Esta experiencia nos debe servir, a nosotros, la nueva generación de futuros médicos jóvenes y también para médicos de mucha experiencia, a no descartar la Historia Clínica, y a no pretender que mediante estudios laboratoriales o imagenológicos nomas podemos diagnosticar pacientes. Los análisis son "complementarios" y como tal, una buena semiología u una detallada historia clínica, a veces, es suficiente.

para la investigación. In OMS; 1993; Ginebra.

5. Manual diagnóstico y estadístico de trastornos mentales (DSM-V). BB: Asociación Americana de Psiquiatría. 2013;(5ta ed. Arlington: APA; ).

6. Rosenberg DA. Web of engaño. Una revista de la literatura del síndrome de Munchausen por poder. Abuso infantil Negl. 1987; 11(4 pg 547-63). 